\title{
XCII. On water waves as asymmetric oscillations and on the stability of free Wave-trains
}

\section{Andrew Stephenson}

To cite this article: Andrew Stephenson (1911) XCII. On water waves as asymmetric oscillations and on the stability of free Wave-trains, Philosophical Magazine Series 6, 21:126, 773-777, DOI: 10.1080/14786440608637094

To link to this article: http://dx.doi.org/10.1080/14786440608637094

册 Published online: 20 Apr 2009.

Submit your article to this journal $\sqsubset \pi$

Џll Article views: 3

Q View related articles $₫$ 
Note.-A. Gockel (Jaltrbuch der Radioaktivität, vii. p. 503, 1910), examined various minerals found by Prof. Strutt to be relatively rich in radium, and obtained radium contents far below his values, accompanied at the same time by unusually high thorium richness. Gockel suggests the possibility of a source of error from the active deposit of thorium, consequent upon the introduction of thorium emanation into the electroscope.

Some experiments were made to test the possibility of such an error affecting the estimation of radium in bodies rich in thorium.

A solution containing as much as $0 \cdot 1$ gram thorianite, having been first boiled to expel radium emanation, was then treated in the same manner as that employed in the estimation of radium. Four experiments were made using both slow and fast admission, in the latter case the gases being admitted into the electroscope as fast as the safety of the gold leaf would permit. The whole of the gases were in this case trunsferred to the electroscope within two minutes subsequent to being cut off from the parent solution. In no case was any certain increase noticed in the rate of collapse of the leaves of the electroscope in three hours after admission.

In view of the fact that no effect was observable in the case of a solution so rich in thorium, it is safe to infer that in no case could the leak of an electroscope be noticenbly affected as read three hours after the slow admission of such quantities of thorium emanation as are associated with amounts of thorium of the order of magnitude dealt with in the estimation of rocks and minerals.

XCII. On Water Waves as Asymmetric Oscillations and on the Stability of Free Wave-1 rains. By Axdrew Stephenson *. 1. W ATER waves furnish a complex oxample of asymmetric oscillations, and it is natural to inquire whether they exhibit the marked energy absorption under direct force of double frequency, which is characteristic of the asymmetric system with one degree of freedom $\uparrow$. The problem is most simply considered as one of steady motion. Direct force may be applied to a deep stream flowing uniformly by a stationary periodic variation of pressure along its surface. Such variation will produce standing waves of equal length. Is this state of motion stable when the wave-length is hall that of the free standing waves?

For the purpose of testing the stability of a train of waves

* Communicated by the Author. + "On a Peculiar Property of the Asymmetric System," Phil. Mag.
Jan. 1911 . 
for small disturbance of a given character, we seek the force system necessary to maintain the disturbance. If the force when acting alone tends continually to damp it, we conclude that the steady motion is unstable for such variation. Thus in the present problem we seek the conditions under which a small standing train of double wave-length, and therefore of nearly free type, may be maintained by pressure variation of the corresponding period in such phase that its action tends to damp out the train without producing other change.

As the discussion involves quantities of different orders of smallness, we shall discard the customary stream and velocitypotential functions in favour of more direct coordinates, thus obtaining equations which are readily applicable to all cases in which the magnitude of the amplitude is involved.

2 . Let $(x, y+\eta)$ be the coordinates of a particle the mean level of which is at distance $y$, positive upwards, from the urdisturbed surface. Then the velocity in the stream-line of mean level $y$ is $c \sqrt{1+\left(\frac{d \eta}{d x}\right)^{2}} /\left(1+\frac{d \eta}{d y}\right)$, where $c$ is a function of $y$, and its horizontal and vertical components are $c /\left(1+\frac{d \eta}{d y}\right)$ and $c \frac{d \eta}{d x} /\left(1+\frac{d \eta}{d y}\right)$.

A displacement $\delta x$ horizontally is given by a displacement along the stream-line of horizontal component $\delta x$, and a vertical displacement $-\frac{d \eta}{d x} \cdot \delta x$. For irrotational motion therefore

$$
\begin{gathered}
\left(\frac{d}{d x}-\frac{\frac{d \eta}{d x}}{1+\frac{d \eta}{d y}} \frac{d}{d y}\right) \frac{c \frac{d \eta}{d x}}{1+\frac{d \eta}{d y}}-\frac{1}{1+\frac{d \eta}{d y}} \frac{d}{d y} \frac{c}{1+\frac{d \eta}{d y}}=0 \\
\text { i. e., } \quad\left(1+\frac{d \eta}{d y}\right)^{2} \frac{d^{2} \eta}{d x^{2}}+\left(1+\left(\frac{d \eta}{d \cdot x^{2}}\right)^{2}\right) \frac{d^{2} \eta}{d y^{2}}-2\left(1+\frac{d \eta}{d y}\right) \frac{d \eta}{d x} \frac{d^{2} \eta}{d x d y} \\
-\frac{1}{c} \frac{d c}{d y}\left(1+\frac{d \eta}{d y}\right)\left(1+\left(\frac{d \eta}{d x}\right)^{2}\right)=0 . \quad \text {. }
\end{gathered}
$$

The pressure is given by

$$
p / \rho=-g \eta-\frac{1}{2} c^{2} \frac{1+\left(\frac{d \eta}{d x}\right)^{2}}{\left(1+\frac{d \eta}{d y}\right)^{2}}+a \text { constant }
$$

4. The forced motion due to a small simple pressure variation of wave-length $\pi / k$ is

$$
\eta=a e^{2 i y} \cos 2 k \cdot r
$$


and the equation of motion for any additional disturbance, $\zeta$, is therefore from (1)

$$
\begin{aligned}
& \frac{d^{2} \zeta}{d x^{2}}+\frac{d^{2} \zeta}{d y^{2}}+4 a k e^{2 k y} \cos 2 k x \frac{d^{2} \zeta}{d x^{2}}+4 a k e^{2 k y} \sin 2 k x \cdot \frac{d^{2} \zeta}{d x d y} \\
& \quad-8 a k^{2} e^{2 k y} \cos 2 k x \frac{d \zeta}{d y}+8 a k^{2} \sin 2 k x \frac{d \zeta}{d x}=0 .
\end{aligned}
$$

Putting

$$
\zeta=\alpha \cos k x+\beta \sin k x,
$$

where $\alpha$ and $\beta$ are functions of $y$, we have from (2)

$$
\begin{aligned}
& \alpha=p\left(e^{k y}+\frac{3}{2} a k e^{3 k y}\right), \\
& \beta=q\left(e^{k y}-\frac{3}{2} a k e^{3 k y}\right) .
\end{aligned}
$$

Now the pressure at the surface is proportional to

which is

$$
-{ }_{c^{2}}^{g} \zeta+\frac{d \zeta}{d y}-6 a k \cos 2 k x \frac{d \zeta}{d y}+2 a k \sin 2 k x \cdot \frac{d \zeta}{d x}
$$

where

$$
-p(a k-\sigma) \cos k x+q(a k+\sigma) \sin k x,
$$

$$
c^{2}=\frac{g}{k}(1+\sigma)
$$

This pressure, being nearly of free period, tends to damp the disturbance if its phase is $-1 / 4$ : that is, if

$$
\eta^{2}(a k-\sigma)=\eta^{2}(a k+\sigma) \text {. }
$$

$|\sigma|$ must therefore be less than $a k$. Thus there is instability for any value of $\sigma$ numerically less than $a k$, the standing disturbance of wave-length $2 \pi /\{k(1+\sigma)\}$ being magnified in one phase $-\tan ^{-1} \sqrt{\frac{a k}{a k}-\sigma}$, and diminished in the numerically equal phase of opposite sign.

Interpreting the result in terms of progressive waves in still water, it is evident that if a periodic pressure variation moves uniformly over the surface, the forced train of equal wave-length constitutes an unstable state of motion if the ratio of the wave-length to that of the free wave of equal speed lies within a range $2 a k$ about the value $1 / 2$; for a small disturbance will result in a series of waves of double wavelength, which is continually magnified through the periodic pressure until the amplitude is large compared with the original motion. The process consists essentially in the continual enlargement of an asymmetric oscillation of approximately free type by a direct force of frequency lying within a range about the double frequency of free oscillation.

For the purposes of experimental illustration it would be simplest to take the case of a stream flowing over a corrugated bed. 
3. Since the free wave of finite amplitude is appreciably asymmetric, it would seem possible from the foregoing that the free train might tend to magnify other relatively stationary trains of nearly the same wave-lengths and relatively smail amplitudes. This question is a partial test of the stability of a free train of waves. To determine the free wave of finito amplitude *, putting

$$
\eta=\alpha \cos k x+\beta \cos 2 k x
$$

where $\alpha$ and $\beta$ are functions of $y$, we find

$$
\begin{aligned}
& \alpha^{\prime \prime}-k^{2} \alpha+\frac{1}{4} k^{2} \alpha^{2} \alpha^{\prime \prime}-\frac{5}{4} k^{2} \alpha \alpha^{\prime 2}-3 k^{2} \alpha \beta^{\prime}-6 k^{2} \alpha^{\prime} \beta-\frac{1}{c} c^{\prime} \alpha^{\prime}=0, \\
& \beta^{\prime \prime}-4 k^{2} \beta=0, \\
& \frac{1}{c} c^{\prime}+2 k^{2} \alpha \alpha^{\prime}=0,
\end{aligned}
$$

subject to the boundary conditions $a=\beta=0$ when $y=-\infty$, and

$$
\begin{aligned}
& \frac{g}{c^{2}} \alpha-2 \alpha^{\prime}+3 \alpha^{\prime} \beta^{\prime}-3 \alpha^{3}+2 k^{2} \alpha \beta-\frac{1}{2} k^{2} \alpha^{2} \alpha^{\prime}=0, \\
& \frac{g}{c^{2}} \beta-\beta^{\prime}+\frac{3}{4} \alpha^{\prime 2}-\frac{1}{4} k^{2} \beta^{2}=0, \\
&
\end{aligned}
$$

when $y=0$.

Hence

$$
\begin{aligned}
& \alpha=u e^{k i y}+{ }_{8}^{5} a^{3} k^{2} e^{3 k y}, \\
& \beta=\frac{1}{2} a^{2} k e^{2 k y}, \\
& c^{2}={ }_{k}^{g}\left\{1+k^{2} a^{2}\left(1-2 e^{2 k i y}\right)\right\} .
\end{aligned}
$$

The equation for the disturbance, $\zeta$, is

$$
\begin{aligned}
& \frac{d^{2} \zeta}{d x^{2}}+\frac{d^{2} \zeta}{d y^{2}}+2 a k e^{k y}\left\{\cos k x\left(\frac{d^{2} \zeta}{d x^{2}}-k \frac{d \zeta}{d y}\right)+\sin k x\left(\frac{d^{2} \zeta}{d x d y}+k \frac{d \zeta}{d x}\right)\right\} \\
& +a^{2} k^{2} e^{2 k y}\left\{\frac{1}{2}\left(\frac{d^{2} \zeta}{d x^{2}}+\frac{d^{2} \zeta}{d y^{2}}\right)+\frac{1}{2} \cos 2 k x\left(5 \frac{d^{2} \zeta}{d x^{2}}-\frac{d^{2} \zeta}{d y^{2}}-8 k \frac{d \zeta}{d y}\right)\right. \\
& \left.\quad+\sin 2 k x\left(3 \frac{d^{2} \zeta}{d x d y}+4 k \frac{d \zeta}{d x}\right)\right\}=0 .
\end{aligned}
$$

* The method is eridently applicable when the depth is finite. In the case of long wares the process is necessarily different. We have then

$$
\eta=\eta_{0}+y\left(\frac{d \eta}{d y}\right)_{0}+\frac{y^{2}}{2 !}\left(\frac{d^{2} \eta}{d y^{2}}\right)_{0}+\frac{y^{3}}{3 !}\left(\frac{d^{3} \eta}{d y^{3}}\right)_{0}
$$

when small quantities of order higher than the second are neglected. Hence, putting $y=-h$, and substituting for the $y$ derivatives from (1) and the surface condition we obtain

$$
\frac{h^{2}}{d x^{2}}\left(\begin{array}{c}
\eta \\
h
\end{array}\right)+\frac{3}{h^{2}}\left\{\left(\frac{g^{h}}{c^{2}}-1\right) \frac{\eta_{0}}{h}+\frac{3}{2}\left(\frac{\eta_{0}}{h}\right)^{2}\right\}=0
$$

the well-known equation for the contours. 
On Water-Waves as Asymmetric Oscillations.

The pressure at the surface is proportional to

$-k\left(1+a^{2} k^{2}\right) \zeta+\left(1+\frac{7}{2} a^{2} k^{2}-3 a k \cos k x-\frac{1}{2} a^{2} k^{2} \cos 2 k x\right) \frac{d \zeta}{d y}+a k \sin k x \frac{d \zeta}{d x}$.

The solution

$$
\begin{aligned}
\zeta= & \left(e^{(k+s) y}+\frac{7}{4} a^{2} k^{2} e^{3 k y}\right) \cos (k+s) x+\frac{9}{8} \alpha^{2} k^{2} e^{3 k y} \cos (k-s) x \\
& +a k e^{(2 k+s) y} \cos (2 k+s) x+a k e^{2 k y} \cos s x-a k e^{|s| y} \cos s x
\end{aligned}
$$

gives surface pressure

and

$$
\left(s-a^{2} k^{3}\right) \cos (k+s) x-a^{2} k^{3} \cos (k-s) x:
$$

$$
\begin{aligned}
\zeta= & \left(e^{(k+s) y}+\frac{7}{4} a^{2} k^{2} e^{3 k y y}\right) \sin (k+s) \cdot x-\frac{9}{8} a^{2} k^{2} e^{3 k y} \sin (k-s) x \\
& +a k e^{(2 k+\delta) y} \sin (2 k+s) x-a k e^{(2 k+s) y} \sin s x-a k e^{|s| y} \sin s . t
\end{aligned}
$$

the pressure

$$
\left(s-a^{2} k^{3}\right) \sin (k+s) x+a^{2} k^{3} \sin (k-s) x .
$$

Hence the disturbance

$$
e^{(k+s) y}\left\{\mathrm{~A} \cos (k+s) x+(\mathrm{C} \sin (k+s) x\}+e^{(k-s) y}\{\mathrm{~B} \cos (k-s) x+\mathrm{D} \sin (k-s) x\}\right.
$$

is maintained by the pressure

$$
\begin{aligned}
& \left\{\left(s-a^{2} k^{3}\right) \mathrm{A}-a^{2} k^{3} \mathrm{~B}\right\} \cos (k+s) x+\left\{\left(s-a^{2} k^{3}\right) \mathrm{C}+a^{2} k^{3} \mathrm{D}\right\} \sin (k+s) x \\
+ & \left\{\left(-s-a^{2} k^{3}\right) \mathrm{B}-a^{2} k^{3} \mathrm{~A}\right\} \cos (k-s) x+\left\{\left(s-a^{2} k^{3}\right) \mathrm{D}+a^{2} k^{3} \mathrm{C}\right\} \sin (k-s) x .
\end{aligned}
$$

The pressure acting alone would tend simply to change the intensity of the disturbance if the two components are proportional to the amplitudes of the trains of corresponding wave-lengths, and if the phases differ by a quarter period from those of the trains; that is, if

$$
\begin{aligned}
& \frac{\left(s-a^{2} k^{3}\right) \mathrm{A}-a^{2} k^{3} \mathrm{~B}}{\mathrm{C}}=-\frac{\left(s-a^{2} k^{3}\right) \mathrm{C}+a^{2} k^{3} \mathrm{D}}{\mathrm{A}} \\
= & \frac{\left(-s-a^{2} k^{3}\right) \mathrm{B}-a^{2} k^{3} \mathrm{~A}}{\mathrm{D}}=-\frac{\left(-s-a^{2} k^{3}\right) \mathrm{D}+a^{2} k^{3} \mathrm{C}}{\mathrm{B}}=q, \text { say. }
\end{aligned}
$$

Hence

$$
q^{2}=-s^{2}
$$

$q$ is therefore always complex, and the free train has no tendency to develop a periodicity of amplitude.

Phil. Mag. S. 6. Vol. 21. No. 126. June 1911. 3 E 